\title{
Management of Edentulous Maxilla with Sagittal-Transverse Deficiency Presenting with Repeated Implant Failures: A Case Report
}

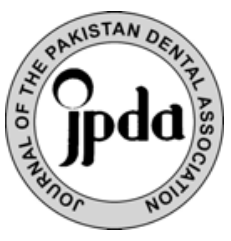

\author{
Muhammad Hasan Hameed ${ }^{1} \quad$ BDS \\ Farhan Raza Khan ${ }^{2} \quad$ BDS, MS, MCPS, FCPS
}

\begin{abstract}
:
Occlusion is an important factor which determines the long term success in dental implantology. Ignoring the occlusion at the treatment planning can lead to poor clinical outcomes. The present report shows a case of an elderly lady presenting with edentulous maxilla which has experienced repeated failure of implants and prostheses. The patient had a deficient maxilla in sagittal and transverse planes. Previous dentists were unable to provide a predictable fixed prosthesis to the patient resulting in patient frustration. However, at the university dental center, the authors took the occlusion into account that not only yielded a functional and esthetic outcome with implant prosthesis but resulted in a satisfied patient as well.
\end{abstract}

KEYWORDS: Dental implant, prosthetics, failure, edentulous maxilla.

HOW TO CITE: Hameed MH, Khan FR. Management of Edentulous Maxilla with Sagittal-Transverse Deficiency Presenting with Repeated Implant Failures: A Case Report. J Pak Dent Assoc 2017; 26(2): 94-97.

Received: 5 January 2017, Accepted: 7 June 2017

\section{INTRODUCTION}

$\mathrm{D}$ ental implant based rehabilitation of the edentulous maxilla is a restorative challenge ${ }^{1}$. Number of variables influence the aesthetic and functional outcomes of the prosthesis. Long term prognosis of implants in the maxilla is less predictable than that of the mandible ${ }^{2}$ Maxillary bone resorption after tooth extraction is more pronounced (both in horizontal and vertical dimensions) than in mandible ${ }^{2}$. Moreover; pneumatization of maxillary sinus leaves little bone for subsequent implant placement $^{3}$. Differential loss of alveolar bone on the facial aspect of maxilla results not only in a cross bite but unwanted occlusal interferences in the subsequent definitive prosthesis ${ }^{2,4}$. This creates mechanical stresses on the dental implants and the associated prostheses ${ }^{4}$.

Implant failure is believed to occur as a consequence of multifactorial process. Various causative factors include surgical trauma, peri-implantitis, smoking, poor bone quantity and/or quality, lack of primary stability, immediate

1. Resident, Operative Dentistry, Aga Khan University, Stadium Road, Karachi, Pakistan

2. Assistant Professor, Dentistry, Aga Khan University, Stadium Road, Karachi, Pakistan

Corresponding author: "Dr. Farhan Raza Khan”

<farhan.raza@aku.edu> loading. The most important factor that is usually overlooked is occlusal trauma and overloading ${ }^{5,6}$.

Occlusion is one of the important factors that determine the long term success of implant-supported prostheses ${ }^{4}$. If occlusion is not taken into account during the treatment planning, marginal bone loss continues to occur which leads to poor treatment outcome and implant failure ${ }^{5}$. Long-term survival and stability of implants requires consideration of proper occlusal scheme and implant-protected occlusion which improves the force distribution. This helps to minimize masticatory overload on bone-implant interfaces especially when occlusal interferences are present ${ }^{4,5}$.

The present case report highlights the importance of implant protected occlusion which must be considered during full mouth rehabilitation of edentulous maxilla with implant supported prostheses.

\section{CASE REPORT}

A 64-year old, hypertensive female presented to the dental clinic at Aga Khan University Hospital with the primary complaint of dislodged metallo-ceramic bridge and exfoliated dental implants. She reported that she had four dental implants placed by a GDP in her upper jaw a year back; the implants started to feel mobile and gradually not only the bridge but two of the implants get dislodged from 

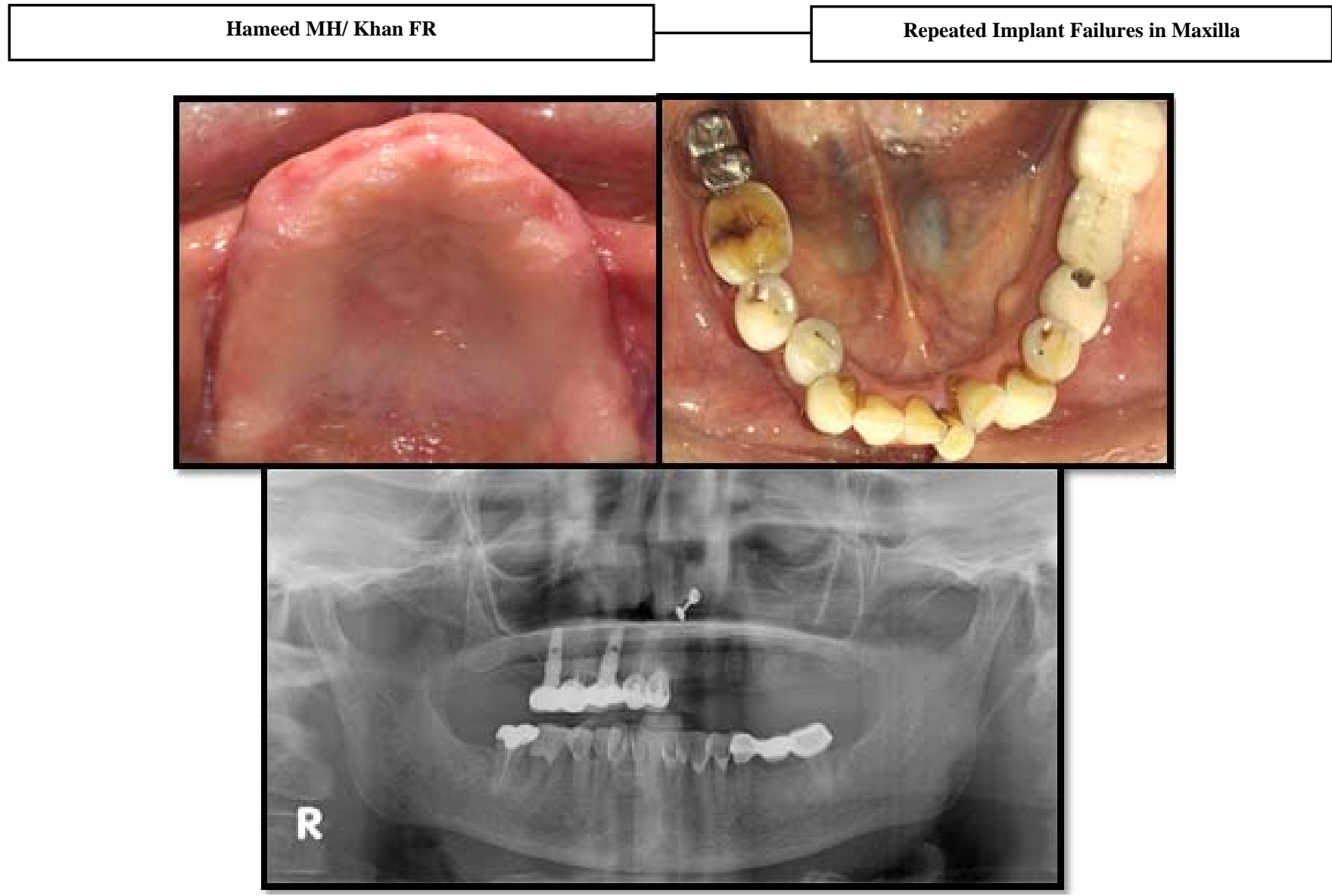

Fig. (1). Pre-operative clinical and radiographic image.

the upper arch and now she is tooth less in the upper arch for over three months.

According to the patient, this was not the first time she had any implant or bridge failed. Her first experience of implant failure was a couple of years back when her upper prosthesis came out with the implants.

She was concerned with the repeated failures and was quite frustrated with the previous dental work. However, getting a removable complete denture was not acceptable for her and she still wanted a fixed solution.

Upon detailed clinical examination, it was seen that the patient had edentulous upper arch and an intact mandibular dentition with fixed metallo-ceramic bridge on \#35-37 and a metal crown on \#47 (Fig. 1). On radiographic examination, two implants were present in positions of \#14 and 16. Patient had class III incisal relationship and a prognathic profile.

The primary challenge in this case was to explore the reasons of repeated failures. We speculate that the most probable explanation for repeated failures in this case was a transversely deficient maxilla \& cross bite in the prosthesis leading to unwanted occlusal interference.
After thorough discussion and counseling, we decided to provide her with implants and bridges. There were following challenges in this plan:

1. Placement of implants in maxilla that is deficient in sagittal and transverse planes

2. Arranging teeth in correct bite versus cross bite or edge to edge relationship

3. Unknown identity of the two retained implants and hence design of the definitive prosthesis

4. What should be surgical protocol and the interim prosthesis?

\section{CASE MANAGEMENT}

After thorough discussion of the management option, informed consent was taken for implants and bridge. We decided to place four new implants and utilize the existing two fixtures so that the definitive prosthesis is supported by six implants. We made a complete denture first and used that as an interim prosthesis (after a little modification) till the time osseo-integration of the newly placed implants is achieved. Her history of repeated failures warranted 
consideration on the occlusal scheme. We planned an edge to edge bite and avoided posterior cross bite and occlusal interference in the definitive bridge.

As she already had two integrated implants placed at the position of \#14 and \#16, the sites favorable for our implants were position of \# 11, 21, 23 and 25. Under local anesthesia, full thickness flap was raised from right to left molar to molar region, with relieving incision at midline frenum. A non-submerged surgical protocol was followed and four Zimmer TSV implants $3.7 \mathrm{mmX} 11.5 \mathrm{~mm}$ were placed with primary stability. Corresponding healing abutments were placed on four new and two old implants (the Zimmer healing abutments were fortunately found to be compatible with her old implants). Incision closed with 3-0 vicryl in simple interrupted fashion.

At two weeks follow-up, we modified the pre-made maxillary complete denture to accommodate and passively house the healing abutments. The denture was delivered and home care instructions including hygiene given to the patient. Following the healing phase after 3 months of implant placement, the patient was called in for her definitive prosthetic phase. At this visit, final polysiloxane putty wash impression was obtained after placing fixture mount transfer abutments (Fig. 2). At subsequent visits, metal casting and bisque trials were done, adjustment made as per the occlusion and esthetic requirements (Fig. 3).

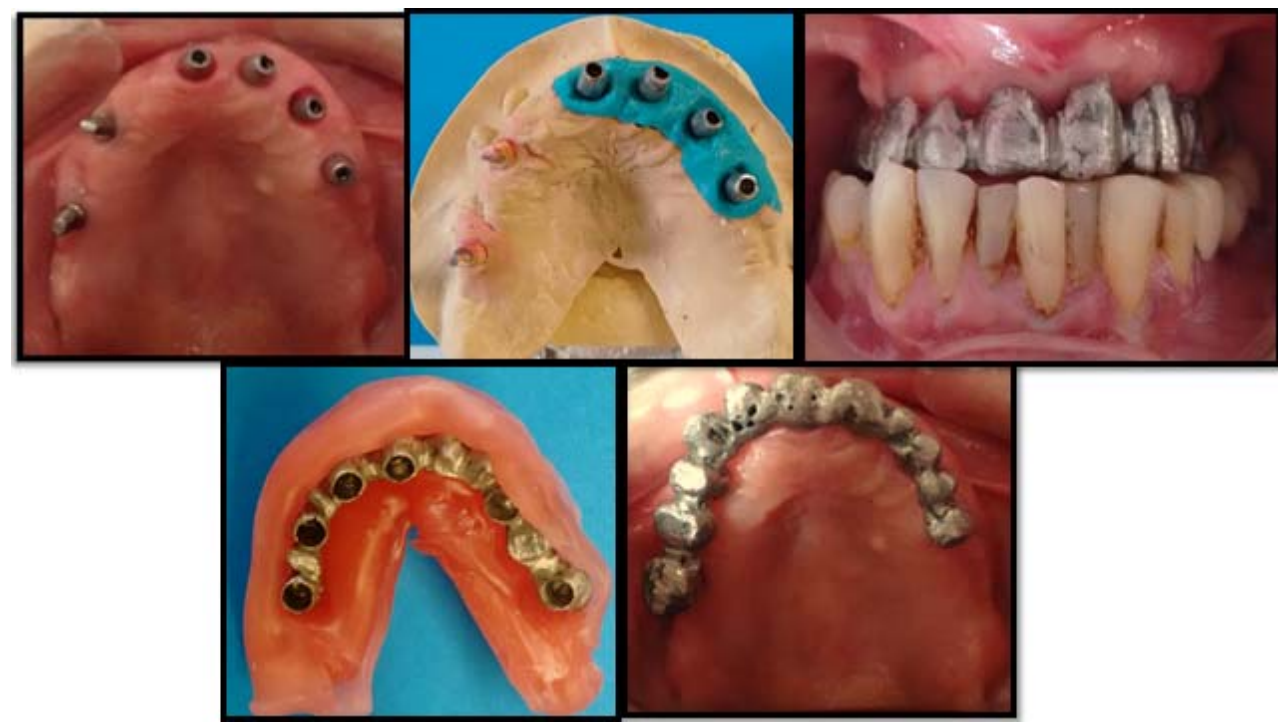

Fig. (2). Appearance of permanent abutments and metal casting trial of the implant prosthesis.

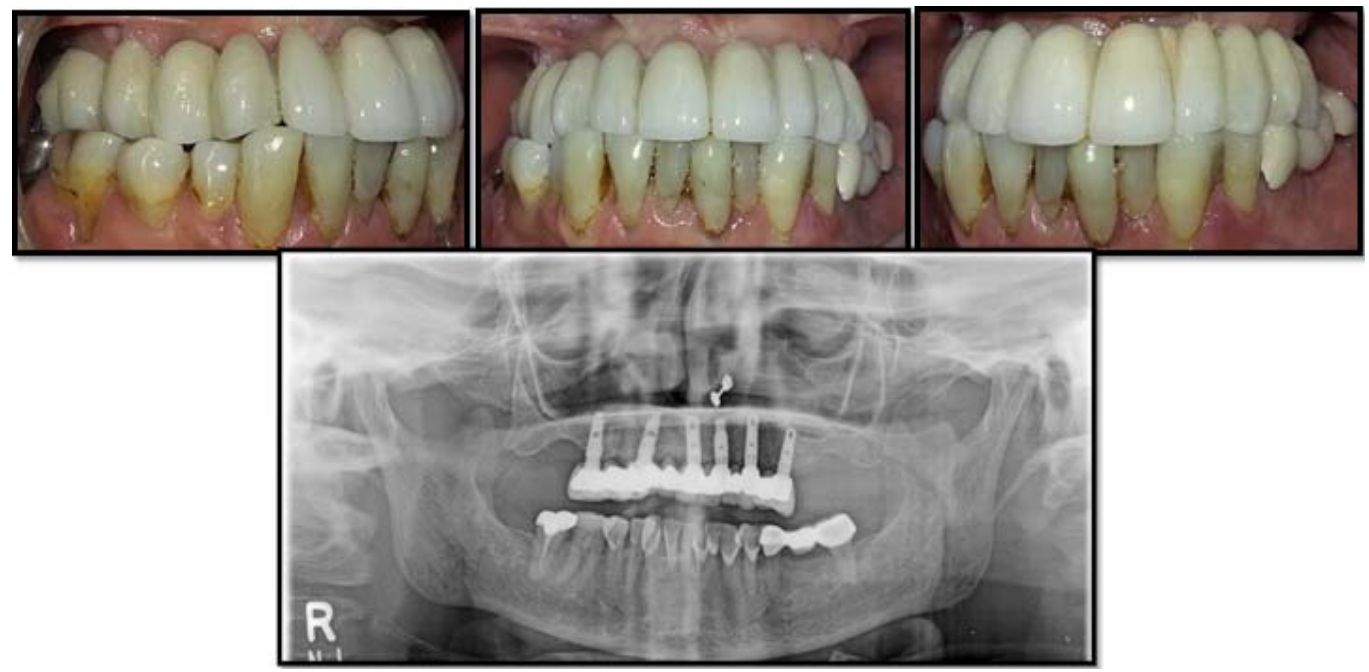

Fig. (3). Post-operative clinical and radiographic image. 
Patient was kept on edge to edge occlusion in order to avoid interferences and premature occlusal contacts. After the patient was fully satisfied with the esthetics, the final prosthesis was delivered to the patient. Patient was monitored on follow-up visits annually. She was pleased with the success of the therapy. To date, there are no detectable clinical or radiographic changes around the implants, and no prosthetic complications have occurred. The patient is scheduled for annual follow- ups to maintain hygiene and periodontal prophylaxis.

\section{DISCUSSION}

This case report delineates a comprehensive treatment planning that can be considered while restoring the edentulous constricted maxilla with implant supported fixed prosthesis. Patients with constricted edentulous maxillae pose multiple challenges because the occlusion of these patients falls into cross bite which could result in occlusal interferences and overloading of implant leading to failure ${ }^{4,7}$. Occlusal overload results in crestal bone loss, thus increasing the anaerobic sulcus depth and predisposes the fixture towards progressive peri-implantitis and eventual loss of implant ${ }^{8,9}$.

Several animal studies have proved that excessive lateral forces from premature occlusal contact can cause excessive marginal bone loss or even osseointegration failure ${ }^{10,11}$. Therefore, in this case, we speculated that occlusal overload from excessive lateral forces acted as one of the factors causing previous implant failures. We managed the case according to the principles of Misch and Bidez ${ }^{12,13}$ who proposed implant-protected occlusion by reducing the occlusal forces on implant prostheses to protect the implants. They suggested that the mechanical stresses on implant can be minimized by adding the number of implants (six or eight instead four or five) or by splinting the implant together to increase the surface of support ${ }^{8,12,13}$. To compensate for the increased magnitude of a force, we increased the surface area of fixtures by increasing the number of implants in the arch and eliminated occlusal interferences on implants keeping the basic principles of implant occlusion which include

1. Anterior guidance whenever possible,

2. Bilateral stability in centric (habitual) occlusion,

3. Wide freedom in centric (habitual) occlusion,

4. Evenly distributed occlusal contacts and forces,

5. No interferences between the retruded position and centric (habitual) position, and

6. Even lateral excursive movements without working and non-working interferences

In our case, we were fortunate that our implant system abutments were compatible with previously placed implants of dissimilar implant systems which helped us in restoring patient's normal occlusion

\section{CONCLUSIONS}

Edentulous maxilla that is deficient in sagittal and transverse planes poses a challenge for implant based prosthetic rehabilitation. Comprehensive and precise evaluation of the patients' needs, followed by appropriate treatment planning provides the restorative dentist with the platform to meet patient expectations. Occlusion is a determining factor for the long term implant success. It must be factored into the treatment planning to obtain a gratifying experience for both the dentist and the patient.

\section{REFERENCES}

1. Bosse LP, Taylor TD. Problems associated with implant rehabilitation of the edentulous maxilla. Dent Clin North Am 1998; 42: 117-27.

2. Jivraj S, Chee W, Corrado P. Treatment planning of the edentulous maxilla. Br Dent J 2006; 201: 261-79; quiz 304.

3. Chiapasco M, Zaniboni M. Methods to treat the edentulous posterior maxilla: implants with sinus grafting. J Oral Maxillofac Surg 2009; 67: 867-71

4. Chen YY, Kuan CL, Wang YB. Implant occlusion: biomechanical considerations for implant-supported prostheses. J Dent Sci 2008; 3: 65-74.

5. Oh TJ, Yoon J, Misch CE, Wang HL. The causes of early implant bone loss: myth or science? J Periodontol 2002; 73: 322-33.

6. Chee W, Jivraj S. Failures in implant dentistry. Br Dent J 2007; 202: 123-9.

7. Schwarz MS. Mechanical complications of dental implants. Clin Oral Implants Res 2000; 11 Suppl 1: 1568.

8. Kim Y, Oh TJ, Misch CE, Wang HL. Occlusal considerations in implant therapy: clinical guidelines with biomechanical rationale. Clin Oral Implants Res 2005; 16: 26-35

9. Lang NP, Wilson TG, Corbet EF. Biological complications with dental implants: their prevention, diagnosis and treatment. Clin Oral Implants Res 2000; 11 Suppl 1: 146-55

10. Miyata T, Kobayashi Y, Araki H, Ohto T, Shin K. The influence of controlled occlusal overload on periimplant tissue. Part 3: A histologic study in monkeys. Int J Oral Maxillofac Implants 2000; 15: 425-31.

11. Isidor F. Loss of osseointegration caused by occlusal load of oral implants. A clinical and radiographic study in monkeys. Clin Oral Implants Res 1996; 7: 143-52.

12. Misch CE, Bidez MW. Implant-protected occlusion. Pract Periodontics Aesthet Dent 1995; 7: 25-9.

13. Misch CE, Bidez MW. Implant-protected occlusion: a biomechanical rationale. Compendium 1994; 15: 13302. 